\title{
Isolated spleen recurrence in a patient with lung adenocarcinoma: A case report
}

\author{
KESATO IGUCHI $^{1}$, OSAMU ISHIBASHI ${ }^{1}$, TADASHI KONDO ${ }^{1}$, KATSUNORI KAGOHASHI ${ }^{2}$, \\ NORIO TAKAYASHIKI ${ }^{3}$ and HIROAKI SATOH ${ }^{2}$ \\ Divisions of ${ }^{1}$ Surgery, ${ }^{2}$ Respiratory Medicine and ${ }^{3}$ Pathology, Mito Medical Center, University of Tsukuba, \\ Mito, Ibaraki 310-0015, Japan
}

Received August 10, 2014; Accepted May 1, 2015

DOI: 10.3892/etm.2015.2524

\begin{abstract}
Spleen metastasis is extremely rare in patients with lung cancer. However, recent improvements in imaging modalities may enable the antemortem diagnosis of spleen metastasis. The present study reports the case of a female patient with lung adenocarcinoma and spleen metastasis. The patient developed isolated spleen metastasis in the postoperative course. This rare metastasis was detected in a follow-up abdominal computed tomography (CT) scan without any symptoms, and was confirmed by fluorodeoxyglucose positron emission tomography (FDG-PET)/CT scan. Although very rare, chest physicians and thoracic surgeons should be alert to the possibility of spleen metastasis development when evaluating the follow-up abdominal CT scan. FDG-PET/CT scanning and an interventional approach should be considered to clarify the possibility of spleen metastasis.
\end{abstract}

\section{Introduction}

In patients with hematological malignancies, the spleen is a common site of involvement $(1,2)$. The incidence of metastasis to the spleen in patients with lung cancer has been reported to be a few percent to $21 \%$ when investigated by autopsy $(3,4)$. By contrast, however, reports of spleen metastasis from lung cancer either at the time of initial diagnosis or during the clinical course are extremely rare (5). In the present study, the case of a patient with lung adenocarcinoma who developed isolated metastasis to the spleen following surgery is described. A review of this rare metastasis in patients with lung cancer was also conducted.

Correspondence to: Professor Hiroaki Satoh, Division of Respiratory Medicine, Mito Medical Center, University of Tsukuba, Miya-machi 3-2-7, Mito, Ibaraki 310-0015, Japan

E-mail: hirosato@md.tsukuba.ac.jp

Key words: fluorodeoxyglucose-positon emission tomography, spleen metastasis, lung cancer

\section{Case report}

A 63-year-old woman presented with the incidental detection of a nodule $30 \mathrm{~mm}$ in diameter in the left lung on chest radiography, at the Mito Medical Center of the University of Tsukuba (Mito, Japan). At the age of 57 years, the patient had undergone a left mastectomy due to breast cancer; however the patient was asymptomatic and had been in good health since then. The physical examination was unremarkable. The chest computed tomography (CT) scan revealed a well-circumscribed mass in the left lower lobe of the lung that measured 30x27x18 mm with specular appearance (Fig. 1). The routine laboratory tests were normal, as were tumor markers including carcinoembryonic antigen. The patient was diagnosed with adenocarcinoma on the basis of pathological examination of transbrochial biopsy specimens. Distant metastasis was not detected. Informed consent was obtained, and the patient underwent lobectomy of the left lower lung and mediastinal lymph node dissection. The final pathological diagnosis was lung adenocarcinoma staged as pT3N2M0, stage IIIB. An EGFR exon 19 deletion was identified. Soon after the surgical resection, the patient received adjuvant postoperative chemotherapy. However, 12 months after the surgery, a follow-up abdominal CT scan revealed three nodular lesions of $\leq 10 \mathrm{~mm}$ in diameter in the spleen (Fig. 2). No metastatic lesions in the liver, adrenal gland and abdominal lymph nodes were found. The splenic nodular lesions were considered to indicate an inflammatory disease such as granuloma. However, fluorodeoxyglucose positron emission tomography (FDG-PET)/CT confirmed uptake in these nodules in the spleen, but there was no other metastatic sites (Fig. 3). The patient then underwent splenectomy. As the resected tumor had the same characteristics as the specimen of the primary lung carcinoma and was positively immunostained for thyroid transcription factor-1 and cytokeratin 7, the tumor was pathologically diagnosed as spleen metastasis from lung adenocarcinoma (Fig. 4). Thereafter, the patient received treatment with four courses of cisplatin and vinorelbine, and was well without any signs of tumor progression.

\section{Discussion}

Although numerous cases of abdominal visceral metastases from lung cancer have been reported $(6,7)$, metastasis to the 


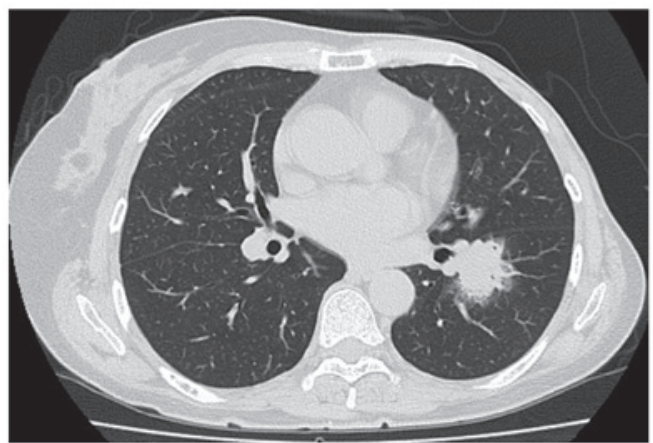

Figure 1. Chest computed tomography revealed a well-circumscribed mass in the lower lobe that measured 34x30x24 mm and had a specular appearance.
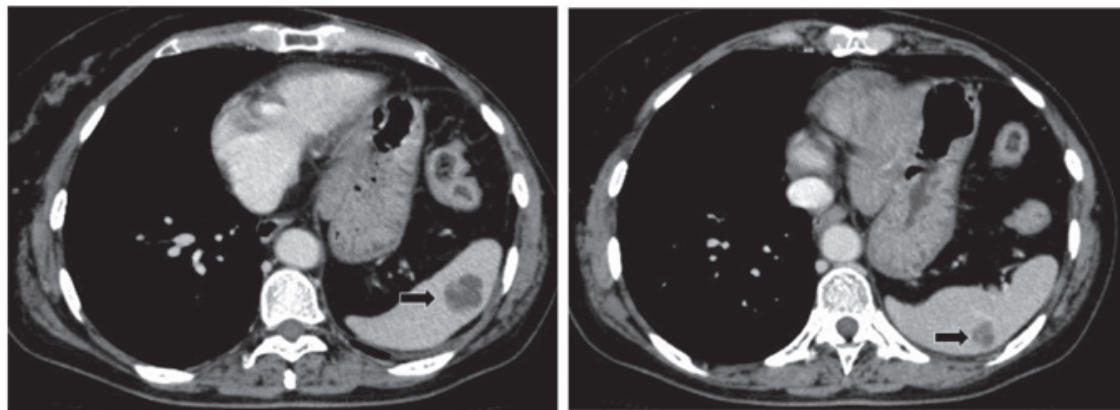

Figure 2. Follow-up abdominal computed tomography scan revealed metastatic lesions of diameter $\leq 15 \mathrm{~mm}$ in the spleen (arrows).
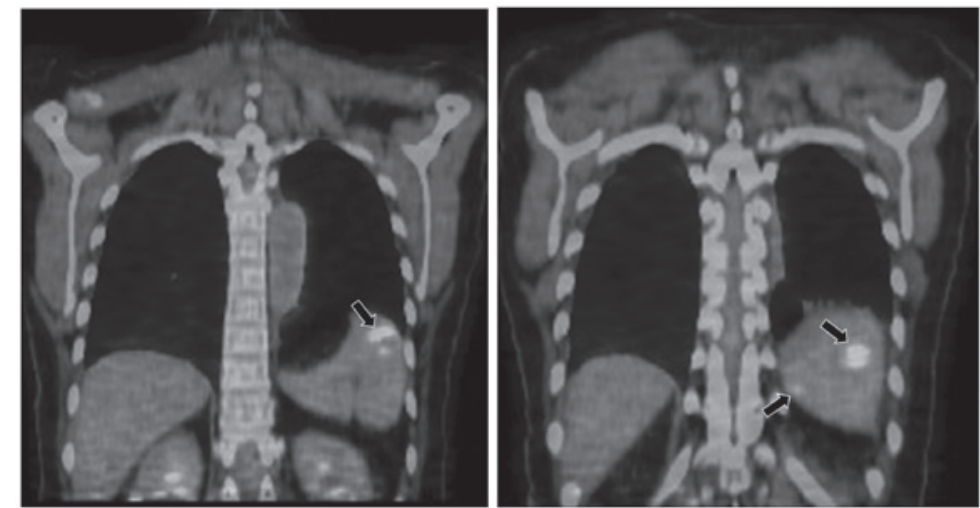

Figure 3. Fluorodeoxyglucose-positron emission tomography/computed tomography confirmed uptake in the nodules in the spleen. There were no other sites of metastasis.
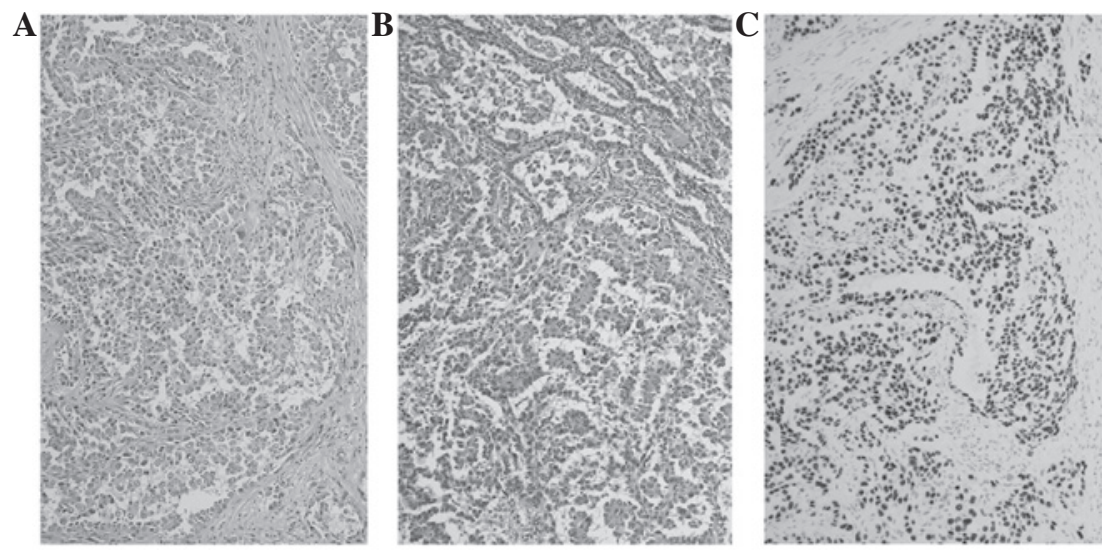

Figure 4. (A) Primary resected lung adenocarcinoma lesion and (B) resected spleen tumor (hematoxylin and eosin staining, x200). (C) Immunostaining of thyroid transcription factor-1 in the resected spleen tumor (hematoxylin and eosin staining, x200). 
spleen from lung cancer is considered to be rare and is usually detected only at autopsy $(8,9)$. The early detection of metastasis to the spleen is challenging since the majority of patients are asymptomatic in the early stage of such metastasis. However, as the availability of CT scanning and ultrasonography has increased, this has enabled spleen metastasis to be diagnosed more accurately (10-12). CT scanning and ultrasonograpy can provide a quick, direct and non-invasive evaluation of spleen metastasis; however, some metastasis cannot be identified by ultrasonograpy because of the effect of air in the stomach and colon. Therefore, abdominal CT scans should be considered for use if spleen metastasis is suspected. Several studies have reported the clinical significance of FDG-PET/CT scanning in the detection of spleen metastasis from lung cancer (13-17). In the present case, FDG-PET/CT was useful for confirming the presence of malignant lesions in the spleen.

We previously evaluated spleen metastasis from lung cancer (18). In the study, 12 (1.2\%) of 997 consecutive patients with lung cancer had spleen metastasis, and isolated spleen metastasis in the absence of widespread disease was considered an extremely rare event (18). All 12 cases had accompanying metastasis to other organs, including the paraaortic lymph nodes, bone, lung and liver, which also suggested a poor outcome for patients with spleen metastasis (18). Moreover, it is noteworthy that all 12 patients exhibited more than one metastatic site in the abdomen. The most common site of metastasis was the lymph nodes in the abdomen. Therefore, it was concluded at that time that spleen mass accompanying metastasis to other abdominal organs in patients with a known lung cancer should be regarded as a metastasis. We recently conducted a review of patients with lung cancer and spleen metastasis, and found 20 such patients (10-15,17,19-30). In these 20 patients, it was notable that all but 4 of the 20 patients had no abdominal metastasis other than spleen metastasis. Therefore, it should now be recognized that spleen metastasis is not necessarily accompanied by abdominal organ metastasis. The difference between our previous study (18) and the recent case reports might be due to the development of imaging modalities and the advancement of knowledge in such rare metastasis.

The incidence of spleen metastasis is considered to differ among histologic types of lung cancer $(3,4,18)$. Small cell lung cancer has been recognized to be the most common cell type likely to metastasize to the spleen and squamous cell lung carcinoma the least likely $(3,4)$. In our previous study of lung cancer metastasis, adenocarcinoma had almost the same frequency of spleen metastasis as small cell lung cancer (1.3 vs. $2.0 \%$, respectively), while in squamous cell carcinoma, only $0.6 \%$ of patients developed spleen metastasis (18). When reviewing recent case reports, however, 5 of the 20 patients with lung cancer and spleen metastasis had squamous cell carcinoma $(12,21,27-29)$. These results indicate that the spleen is a possible site to which disease may spread in patients with advanced or recurrent lung cancer, including those with squamous cell carcinoma of the lung.

With regard to the radiological classification of spleen metastasis dependent on pathological examination, there may be four types, known as solitary, multiple, microscopic and diffuse. Sardenberg et al presented three main macroscopic patterns: Macronodular, micronodular and diffuse (17). The latter two types might not be apparent in abdominal CT scans, although FDG-PET/CT scanning may detect the diffuse type of spleen metastasis. In our previous study, the solitary nodular type was the most common, and in a review of recent case reports, the solitary type was confirmed as the most common type $(10,12-17,19-21,23,26)$. The differential diagnosis of a solitary nodule or nodules in the spleen includes several diseases, either benign or malignant (31). In differential diagnosis, FDG-PET/CT scanning can be useful to identify whether the lesion is malignant (13-17).

A mass in the spleen accompanied by metastasis to other abdominal organs in a patient known to have lung cancer or with a history of lung cancer should be regarded as a metastasis. In addition, an isolated splenic mass in the absence of abdominal metastatic disease elsewhere should also be considered likely to represent a metastasis. An isolated spleen mass identified at the initial diagnosis or during the clinical course in a patient with lung cancer should be considered for an interventional approach and can provide pathological material.

\section{References}

1. Kraemer BB, Osborne BM and Butler JJ: Primary splenic presentation of malignant lymphoma and related disorders. A study of 49 cases. Cancer 54: 1606-1619, 1984.

2. Sharpe RW, Rector JT, Rushin JM, Garvin DF and Cotelingam JD: Splenic metastasis in hairy cell leukemia. Cancer 71: 2222-2226, 1993.

3. Auerbach O, Garfinkel L and Parks VR: Histologic type of lung cancer in relation to smoking habits, year of diagnosis and sites of metastases. Chest 67: 382-387, 1975.

4. Densert $\mathrm{O}$ and Söderström J: Diffuse metastases in bronchial cancer. Acta Pathol Microbiol Scand 64: 477-484, 1965.

5. Morgenstern L, Rosenberg J and Geller SA: Tumors of the spleen. World J Surg 9: 468-476, 1985.

6. Abrams HL, Spiro R and Goldstein N: Metastases in carcinoma; analysis of 1000 autopsied cases. Cancer 3: 74-85, 1950.

7. Warren S and Gates O: Lung cancer and metastasis. Arch Pathol 78: 467-473, 1964.

8. Hirst AE Jr and Bullock WK: Metastatic carcinoma of the spleen. Am J Med Sci 223: 414-417, 1952.

9. Klein B, Stein M, Kuten A, et al: Splenomegaly and solitary spleen metastasis in solid tumors. Cancer 60: 100-102, 1987.

10. Edelman AS and Rotterdam H: Solitary splenic metastasis of an adenocarcinoma of the lung. Am J Clin Pathol 94: 326-328, 1990.

11. Imada $H$, Nakata $H$ and Horie A: Radiological diagnosis of splenic metastasis and its prevalence at autopsy. Nihon Igaku Hoshasen Gakkai Zasshi 51: 498-503, 1991 (In Japanese).

12. Kinoshita A, Nakano M, Fukuda M, et al: Splenic metastasis from lung cancer. Neth J Med 47: 219-223, 1995.

13. Yen RF, Wu YW, Pan MH and Tzen KY: Early detection of splenic metastasis of lung cancer by ${ }^{18} \mathrm{~F}$-2-fluoro-2-deoxyglucose positron emission tomography. J Formos Med Assoc 104: 674-676, 2005.

14. Fujii M, Tanaka H, Sawazumi T, et al: A case of solitary splenic metastasis following operation for pulmonary pleomorphic carcinoma: Detected at an early stage by FDG-PET. Nihon Kokyuki Gakkai Zasshi 46: 950-954, 2008 (In Japanese).

15. Tang H, Huang H, Xiu Q and Shi Z: Isolated splenic metastasis from lung cancer: Ringleader of continuous fever. Eur Respir Rev 19: 253-256, 2010.

16. Soussan M, Pop G, Ouvrier MJ, Neuman A and Weinmann P: Diagnosis of synchronous isolated splenic metastasis from lung adenocarcinoma: Complementary role of FDG PET/CT and diffusion-weighted MRI. Clin Nucl Med 36: 707-709, 2011.

17. Sardenberg RA, Pinto C, Bueno CA and Younes RN: Non-small cell lung cancer stage IV long-term survival with isolated spleen metastasis. Ann Thorac Surg 95: 1432-1434, 2013.

18. Satoh H, Watanabe K, Ishikawa H, Yamashita YT, Ohtsuka M and Sekizawa K: Splenic metastasis of lung cancer. Oncol Rep 8: 1239-1241, 2001.

19. Scintu F, Carta M, Frau G, Marongiu L, Pipia G and Casula G: Splenic metastases of pulmonary carcinoma. Apropos of a clinical case. Minerva Chir 46: 1277-1280, 1991 (In Italian). 
20. Tomaszewski D, Bereza S and Sternau A: Solitary splenic metastases from lung cancer - one-time surgical procedure. Pneumonol Alergol Pol 71: 533-537, 2003 (In Polish).

21. Pramesh CS, Prabhudesai SG, Parasnis AS, Mistry RC and Sharma S: Isolated splenic metastasis from non small cell lung cancer. Ann Thorac Cardiovasc Surg 10: 247-248, 2004.

22. Lachachi F, Abita T, Durand Fontanier S, Maisonnette F and Descottes B: Spontaneous splenic rupture due to splenic metastasis of lung cancer. Ann Chir 129: 521-522, 2004 (In French).

23. Schmidt BJ and Smith SL: Isolated splenic metastasis from primary lung adenocarcinoma. South Med J 97: 298-300, 2004.

24. Assouline P, Leger-Ravet MB, Paquet JC, et al: Splenic metastasis from a bronchial carcinoma. Rev Mal Respir 23: 265-268, 2006 (In French).

25. Sánchez-Romero A, Oliver I, Costa D, et al: Giant splenic metastasis due to lung adenocarcinoma. Clin Transl Oncol 8: 294-295, 2006
26. Van Hul I, Cools P and Rutsaert R: Solitary splenic metastasis of an adenocarcinoma of the lung 2 years postoperatively. Acta Chir Belg 108: 462-463, 2008

27. Ando K, Kaneko N, Yi L, et al: Splenic metastasis of lung cancer. Nihon Kokyuki Gakkai Zasshi 47: 581-584, 2009 (In Japanese).

28. Chloros D, Bitzikas G, Kakoura M, Chatzikostas G, Makridis C and Tsitouridis I: Solitary splenic metastasis of squamous lung cancer: A case report. Cases J 2: 9091, 2009.

29. Dias AR, Pinto RA, Ravanini JN, Lupinacci RM, Cecconello I and Ribeiro U Jr: Isolated splenic metastasis from lung squamous cell carcinoma. World J Surg Oncol 10: 24, 2012.

30. Oussama B, Makrem M, Neji FM, et al: Non small cell lung cancer revealed by a solitary splenic metastasis of lung cancer. Tunis Med 91: 484-485, 2013 (In French).

31. Compérat E, Bardier-Dupas A, Camparo P, Capron F and Charlotte F: Splenic metastases: Clinicopathologic presentation, differential diagnosis, and pathogenesis. Arch Pathol Lab Med 131: 965-969, 2007. 